\title{
Performance Modelling of Peer-to-Peer Routing
}

\author{
Idris A. Rai, Andrew Brampton, Andrew MacQuire and Laurent Mathy \\ Computing Department, \\ Lancaster University \\ \{rai,brampton,macquire,laurent\}@ comp.lancs.ac.uk
}

\begin{abstract}
We propose several models based on discrete-time Markov chains for the analysis of Distributed Hash Tables (DHTs). Specifically, we examine the Pastry routing protocol, as well as a Stealth DHT adaptation of Pastry to compute their exact expressions for average number of lookup hops. We show that our analytical models match with the protocols' simulation results almost perfectly, making them ideal for rapid evaluation.
\end{abstract}

\section{Introduction}

Peer-to-peer routing has now been used for several years in a diverse range of applications. Despite the age of many such protocols, little work has gone into providing their formalised models. Existing analysis typically consists of simulated network scenarios rather than any proven mathematical models. While this is not necessarily the case for older, unstructured algorithms, it is certainly true for newer, structured protocols. As compared to simulations, models can allow for much quicker evaluation of protocols at a wide range of settings. Furthermore, they can sometimes help to gain an in-depth understanding of the protocols. Given the popularity of many such peer-to-peer systems, it is therefore important to provide their formalised models.

The approach to routing in most Distributed Hash Table (DHT) based peer-to-peer systems involves iteratively or recursively forwarding a message closer to its eventual destination based on local knowledge at each node, reducing the number possible recipients with each hop. Consequently, most protocols (e.g. Pastry, Tapestry, Chord and CAN $[6,10,9,5]$ offer an expected $O(\log N)$ number of lookup hops as an upper bound. In many of these previous works, this represents the extent to which the proposed sys-

1-4244-0910-1/07/\$20.00 (C)2007 IEEE. tems are mathematically modelled; all remaining study is based upon simulation or implementation results.

In this paper, we aim to address the lack of formal DHT mathematical analysis by modelling DHT routing protocols using discrete-time Markov chains to compute the expected number of lookup hops. Specifically, we consider the Pastry protocol [6] and a "Stealth DHT" adaptation of Pastry [1]. As Pastry was one of the first DHT protocols to be proposed, it provides a suitably general representation of DHT routing that we also describe in greater detail in Section 2. Conversely, our previously proposed Stealth DHT work is a recent development, allowing for unreliable nodes to be separated from core DHT routing at a low cost, as explained further in Section 5.

We know of only one other work that mathematically analyses the performance of DHT protocols [7]. This work proposes a formal framework based on Markov chains to prove the performance of routing protocols in BaRT [8] and Koorde [3]. Although the analytical approach taken by Spognardi et al. also uses Markov chains, the differences in routing methods between these protocols and Pastry make it impossible to model Pastry and its associated Stealth DHT using the exact methodology as proposed in [7].

We first study Perfect Routing models, wherein we assume that an intermediate node along a routing path always finds the "correct" next hop for a message. In practice, however, this is unrealistic; actual routing tables are usually incomplete, with several empty cells. To counter this, we derive other models for the protocols that emulate this imperfection: Models with Imperfect Routing. We define a route as "failed" if a node does not forward a message via the next expected node (e.g. it uses a entry that is closer to the destination but which shares the same prefix-match length as itself with respect to the target ID). These models allow us to derive the exact expressions for the number of average hops required to reach any node in the DHT (i.e. the lookup length).

We validate our models using simulations of the Pastry and Stealth DHT protocols, finding that there is a good 
match between simulation results and the models. Since simulations provide a realistic example of imperfect routing tables, the good validation results show that the models formally prove the routing performance of the protocols. Therefore, the expressions of the average number of hops obtained through the models can be directly used instead of simulations to quickly evaluate the protocols. Moreover, the model results show that the increase in routing imperfection exponentially affect the lookup length of the protocols. The results from the models also help to improve understanding in the choice of Pastry's inherent configuration parameter $b$, as defined in the following section.

The remainder of this paper is organized as follows: We present an overview of Pastry in Section 2. In Section 3 we discuss the Pastry model with perfect routing and derive the expressions for the average number of lookup hops. We then discuss the Pastry model with imperfect routing and validate the model using simulations in Section 4 . We model Stealth DHT routing performance and validate the models in Section 5 before we finally conclude the paper in Section 6.

\section{Pastry Overview}

Each node on a Pastry network has a unique identifier (ID), randomly generated within the address space. The address space is dynamically partitioned into regions with each region being assigned to the single node whose ID is closest. Node IDs are represented in base $2^{b}$ where $b$ is a constant representing the number of bits in each digit of ID. Each node maintains a routing table, which is conceptually a $\log _{2^{b}} N \times 2^{b}$ array, where $N$ is the size of the address space. Thus each row of the array is partitioned into $2^{b}$ cells, which can accommodate more than one entry. The dimensions of the routing table array are so given because the entries in a row $n$ contain references to nodes whose IDs share a common prefix of length $n$ digits. The first row is conceptually row 0 containing entries that have no prefix match, and since there are only $\log _{2^{b}} N$ rows it is impossible to have all digits in common.

The routing procedure for a node that sends or forwards a message is to select the row of its routing table corresponding to its prefix match with the destination ID and pick as a next hop the entry of the column corresponding to the value of the first (non-matching) digit of the destination ID. For example if a message arrives and the destination ID has $n$ prefix matches, the next node will be referenced in the $n^{t h}$ row and in the $(n+1)^{t h}$ digit's column. This ensures that the next hop of the message shares a longer ID prefix with the destination than the current node (and is therefore closer to the destination). It should be clear that one column per row of the routing table contains an empty entry: this is the column corresponding to the $n^{\text {th }}$ digit of the ID of the node holding the routing table (i.e. the node itself). This is because the corresponding entry in row $n$ would then share a prefix of length $n+1$ with the node, and should therefore belong on the following row. This very concise and simplified description of the routing procedure is sufficient for our discussion and we refer the reader to [6] for further details of Pastry routing.

The maximum number of hops per message for a Pastry network of $N$ nodes is given as $\log _{2^{b}} N$. This expression is obtained because, in Pastry, routing follows a path governed by a balanced $2^{b}$-ary tree that spans the entire name space. The $2^{b}$-ary tree is formed due to the structure of the routing tables, where each node is a source for such a tree.

In a $2^{b}$-ary tree, the network population is reduced by a factor of $2^{b}$ each hop until the lookup message reaches the destination node. The number of hops a message takes, $h$, is thus obtained as: $N / 2^{(b h)}=1$, which leads to $h=\log _{2^{b}} N$.

Therefore $h$ is the number of hops when each node along the path improves the lookup path towards the destination by exactly one prefix match, which is an ideal case. We call the expression $h$ the Log Model for Pastry. In practice however, there is a chance of a node's ID improving the match by more than one prefix, or a node may not improve the prefix match at all (failed routes). In the former case, it is obvious to see that the actual average number of hops per message in Pastry is less than $h$.

Despite this, the Log model has been used to verify Pastry routing performance before $[6,1]$. We found, however, that the use of the Log model to validate simulation results depends on the input parameters used in simulators such as leafset size. In this paper, we model Pastry and Stealth DHT routing protocols to derive the exact expressions for the average number of lookup hops.

\section{Pastry Model with Perfect Routing}

In this section we consider an ideal Pastry routing protocol wherein a node always forwards a message to the next hop that matches the key by at least one prefix more than itself. Recall that an ID's digits are represented in base $2^{b}$. Therefore, the probability that two randomly chosen IDs share a single prefix is $p=1 / 2^{b}$. Thus, the probability that two randomly chosen IDs do not to share any prefix is $q=\frac{2^{b}-1}{2^{b}}$

A Pastry lookup (routing path) is made up of all nodes that participate to deliver the message including the source and destination nodes. We model the protocol using a discrete-time Markov chain where each state represents the number of prefix matches a particular node shares with the destination. Each node on a routing path is thus modelled by a state. Let $X_{n}$ be a state of the Markov chain at a time $n$. Pastry routing can be modelled using $h+2$ states such that $X_{n} \in\{0,1,2, \cdots h, h+1\}$, where $h$ is the maximum 


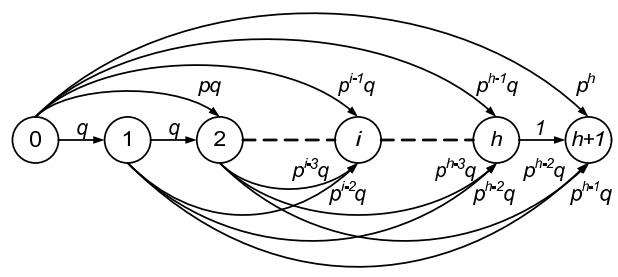

Figure 1. Markov Chain without failures

number of hops a message can take. A state $X_{n}=0$ is also called the source state, where a node has a key to lookup from the network. Conversely, state $X_{n}=h+1$ is the destination state. Before sending a message, a source node may already be at any state. For instance, it can be at state $h+1$ if its ID matches that of the key, or at state $i$ if it shares $i-1$ prefixes with the destination. At state $X_{n}=0$, a node only compares its ID to that of the key to identify the next node to forward the message to (the next state). At any other state $i<h+1$, upon receiving a message to forward, a node finds the next hop from its routing table.

The structure of routing tables, when they are full, guarantees each node on the lookup path to improve the routing towards the destination by one prefix match. In addition, nothing stops a node having more than one prefix match with the target. This however, is not guaranteed and happens only by chance. Therefore, the probability of improving a route by exactly one prefix match is equal to the probability that the next digit following the guaranteed one in the next hop ID does not match the corresponding digit of the destination ID. From our previous discussion, this probability is $q$.

We denote transition probability from state $i$ to state $j$ as $p_{j i}=P\left(X_{n}=j / X_{n-1}=i\right)$. From the routing discussion above, we can see that transition probabilities from state $i$ to state $j\left(p_{j i}\right)$ always exist for all $j>i$. Note that $p_{j i}$ for $j>i+1$ represent transition probabilities when a node is fortunate enough to improve the routing by more than one prefix match. The perfect routing protocol ensures that a key gets closer to the destination every time the message is relayed to another node, then for $j \leq i$, the conditional probability is:

$$
p_{j i}=0 \quad \forall j \leq i
$$

Generally, a transition from state $i$ to state $j$ occurs when a node improves the routing by $j-i$ prefixes, (i.e., the guaranteed prefix match and $j-i-1$ extra matches that could happen by chance). Thus, the transition leads to the following corresponding expression of transition probability:

$$
p_{j i}=p^{j-i-1} q, \quad \forall j>i
$$

where $q=1-p$.

Recall that the perfect routing model assumes that nodes and routes do not fail, which means that $p_{j j}=0$. This

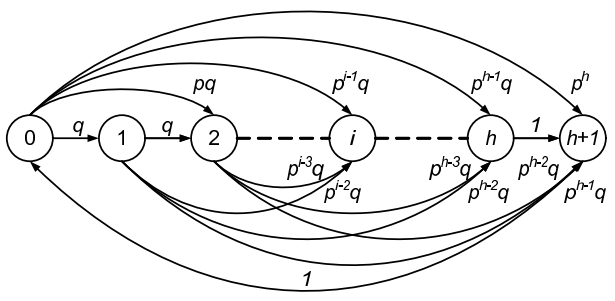

Figure 2. Modified Markov Chain for Pastry routing without failures

allows us to get the general expression for transition probabilities as follows:

$$
p_{j i}= \begin{cases}0 & \text { if } j \leq i \\ p^{j-i-1} q & \text { if } i<j \leq h \\ 1 & \text { if } i=h, j=h+1 .\end{cases}
$$

Fig. 1 shows the Markov chain for the perfect Pastry routing model. Observing the figure, one can note that all states of the chain are transient except the last state, which is absorbing since once entered, the chain never leaves it. As a result, the chain does not exhibit irreducible and aperiodic properties necessary to obtain steady state, stationary distributions. Therefore, the average number of lookup hops, which is obtained from the mean recurrence time of state $h+1$, cannot be computed from the Markov chain. To be able to derive the average number of lookup hops, we transform the chain to an irreducible and aperiodic Markov chain by adding a sure transition from state $h+1$ to state 0 as seen in Fig. 2.

The transition probability matrix for the Pastry routing model corresponding to Fig. 2 is:

$$
\mathbf{P}=\left(\begin{array}{ccccccc}
0 & q & p q & p^{2} q & \cdots & p^{h-1} q & p^{h} \\
0 & 0 & q & p q & \cdots & p^{h-2} q & p^{h-1} \\
0 & 0 & 0 & q & \cdots & p^{h-3} q & p^{h-2} \\
\cdots & \cdots & \cdots & \cdots & \cdots & \cdots & \cdots \\
0 & 0 & 0 & 0 & \cdots & q & p \\
0 & 0 & 0 & 0 & \cdots & 0 & 1 \\
1 & 0 & 0 & 0 & \cdots & 0 & 0
\end{array}\right)
$$

The solution of the chain, which is the steady state probabilities, is the normalised solution of the following system of linearly dependent equations with variable vector $\mathbf{x}=\left(x_{0}, x_{1}, \cdots, x_{h+1}\right)$

$$
\mathrm{xP}=\mathrm{x}
$$

After some simple arithmetic operations and inspection of the transition matrix, it can be shown that the solution to the above system of linear equations is given as:

$$
x_{i}= \begin{cases}x_{h+1} & \text { if } i=0 \\ q x_{0} & \text { if } i=1 \\ x_{i-1} & \text { if } 1<i \leq h\end{cases}
$$


The solution of the system is then given as

$$
\pi_{i}=\frac{x_{i}}{\sum_{j=0}^{h+1} x_{j}}, \text { for } 0 \leq i \leq h+1 .
$$

It can be easily shown that

$$
\sum_{i=0}^{h+1} x_{i}=2 x_{0}+h q x_{0}
$$

which implies that

$$
\pi_{h+1}=\frac{1}{2+h q}
$$

From Leon-Garcia [4], the mean recurrence time for state $h+1$ is given as:

$$
\begin{aligned}
E\left[T_{h+1}\right] & =\frac{1}{\pi_{h+1}} \\
& =2+h q
\end{aligned}
$$

The average number of hops for a lookup to reach a destination is the average number of transitions to move state 0 to state $h+1$, which is equivalent to the mean recurrence time of state $h+1$ minus the transitions that do not represent hops, i.e., from state 0 to all other states and from state $h+1$ to state 0 . Recall that transitions from state 0 are not hops as they only represent a chance prefix match the source may have with the target, and the transition from state $h$ to state 0 does not represent a hop and is added only to obtain stationary probabilities of the Markov chain. The aggregate expected probability for these two cases of transitions is equivalent to $\sum_{i=1}^{h+1} p_{i 0}+p_{0, h+1}$, which is 2 . We therefore obtain the average number of hops for Pastry with perfect routing $\left(H_{\text {pastry }}\right)$ as follows:

$$
\begin{aligned}
H_{\text {pastry }} & =E\left[T_{h+1}\right]-2 \\
& =h q
\end{aligned}
$$

Note that the comparison between the average routing performance of the Pastry model with perfect routing (Equation (7)) and the Log model $\left(\log _{2^{b}} N\right)$ is a function of $q$, which is a protocol configuration parameter $b$. The value of $b=4$ has been typically used in Pastry [6]. Using the derived expression for the average number of hops, we can determine how the performance difference between the Log model and the Pastry model depends on $b$ values. Particularly, the ratio of the average number of hops of the Log model and the Pastry model without failures is $1 / q=\frac{1}{\left(1-1 / 2^{b}\right)}$. Note that $\lim _{b \rightarrow \infty} 1 / q \rightarrow 1$, which means that the model asymptotically converges quickly to the upper bound. This shows the extent to which the average number of hops between the two models compares as $b$ varies, which can aid in deciding on an appropriate value of $b$. From this, we can conclude that small values of $b$ are the superior options.

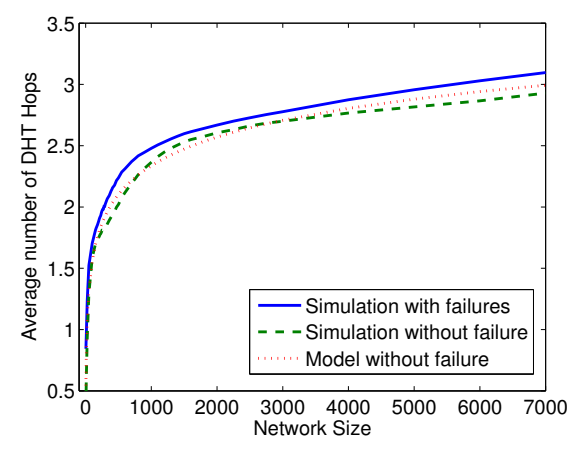

\section{Figure 3. Validation of Pastry model without routing failures}

\subsection{Validation}

We validate our models against simulations that were carried out using our own discrete-event packet-level Pastry simulator, based on Pastry [6]. In [1], we showed that our simulator can be validated against Microsoft's Pastry Version 3.0A simulator, as well as a real world implementation. Each network size was simulated at least five times on a famous GT-ITM [2] generated transit-stub topology of 1,000 routers, with $4 \%$ transit nodes. DHT nodes, whose numbers we vary from 10 to 7,000, were connected to this topology in a random fashion. In each simulation run, 10,000 messages (each with a randomly generated key) were sent from a randomly selected source.

Fig. 3 shows the average number of hops for the perfect routing model and for the simulations, both with and without failures. The latter is obtained by considering only messages that were delivered without routing failures. We clearly see that the results for the Pastry model with perfect routing matches well against the simulation results of Pastry without failures. However, the model underestimates the simulation results with failures.

By definition, the perfect routing model assumes that no route between nodes fails. That is, a node in the perfect routing model always finds the correct next hop reference in its routing table, which is possible only if routing tables are full. Full routing tables further imply that all nodes along a routing path use their routing tables to identify the next hop (except the node just before the destination). However, note that $p_{h+1, h}=1$ indicates that the next hop for the last hop can either be determined using routing tables or leafsets.

Realistically, available routing table population mechanisms in Pastry do not guarantee complete routing tables, and neither is this required for the protocol to work. It is often the case that some cells in a routing table are empty. We therefore next model Pastry routing performance taking into account this routing imperfection. 


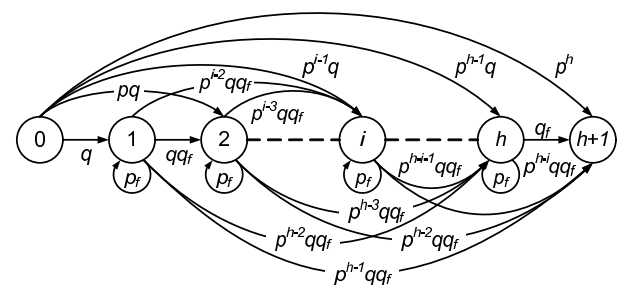

Figure 4. Markov Chain with routing failures

\section{Pastry model with imperfect routing}

We say a route between two nodes fails if a node finds that the cell for the expected correct reference to the next hop is empty. When a route fails, a forwarding node has to look for another next hop reference from other cells in its routing table. The new next hop reference is often chosen as the closest node to the destination on the same row of the routing table as the missing entry. Therefore, it does not improve the lookup towards the destination because, except for the entries in the correct cell, all entries on the same row share the same number of prefixes to the key as the node holding the routing table. From a Markov-chain perspective, this means that the state of the chain is unchanged. The same applies if the failed route occurs at the source of the message.

We assume that a message is equally likely to fail at any state with probability $p_{f}$. Transition probabilities for the model with failures are obtained in a similar way as the model without failures and are summarised as follows:

$$
p_{j i}= \begin{cases}0 & \text { if } j<i \\ p_{f} & \text { if } j=i, i \neq 0, h+1 \\ p^{j-i-1} q & \text { if } i=0, \forall j>i \\ p^{j-i-1} q q_{f} & \text { if } i \geq 1, i<j \leq h \\ q_{f} & \text { if } i=h, j=h+1 .\end{cases}
$$

Fig. 4 shows the Markov chain for the model. Note that routes do not fail at states 0 and $h+1$. Recall that state 0 is the state used to decide the source's beginning state on the chain, and state $h+1$ is the destination.

Using the same procedures as used for the Pastry model with perfect routing, we obtain the average number of lookup hops for Pastry routing model with failures as:

$$
H_{\text {pastry }}^{f}=h \frac{q}{q_{f}}
$$

where $p_{f}$ is the probability of route failure and $q_{f}=1-$ $p_{f}$. We next discuss validation results of the model using simulations of the protocols.

\subsection{Validation}

Before presenting the validation results, we first discuss the various methods we used to compute the probability

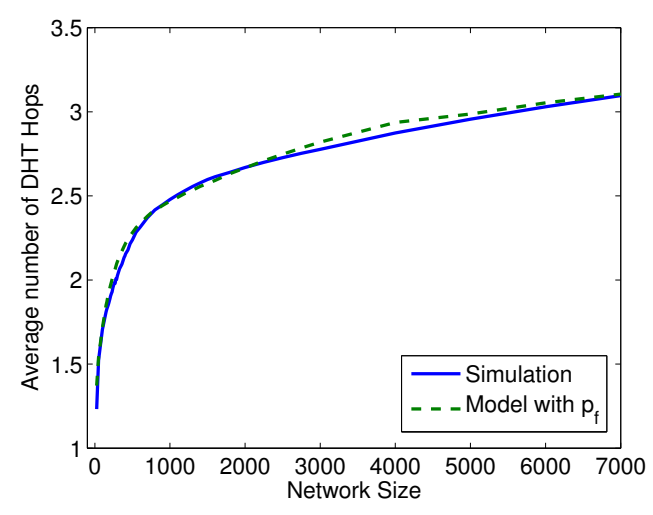

Figure 5. Validation of Pastry model with rout-
ing failures

of route failures. The correct approach is to analytically compute the probability of failures based on network input workload. As our efforts in this regard proved futile, we resorted to the use of simulation results. There are two possible ways this can be done: one is to use the fraction of empty cells per row in a routing table, the other involves tracking down all hops that are due to failed routes. We considered the latter option over the former as it provides the actual failures from the simulation. As such, we tracked all instances in the simulator whereby a node fails to find a next hop reference in its routing table. If we denote the number of failed routes from the simulations, the total number of messages, and the average number of hops for simulations as $F_{r}, M$, and $H_{\text {sim }}$ respectively, then, for each network size, we compute the probability of route failure per state as:

$$
p_{f}=\frac{F_{r}}{H_{\text {sim }} M h}
$$

Where $h$ is the number of states where route failures are possible. In some cases a fixed probability $\left(\overline{p_{f}}\right)$ of route failure per state for each network size computed as the mean of probabilities obtained from simulations as given in Equation (9) offers good validation results.

Fig. 5 shows the average number of hops for the model against the simulation results as a function of network size. We observe that simulations match the results of the model very well when $p_{f}$ as given in Equation (9) is used.

It should be noted that both approaches of computing the probability of route failure per state result in some estimation of failure probabilities only. Moreover, the assumption that the probability of a route failure is the same for each hop is not realistic. Simulation results show that a large fraction of route failures occur at a single state. To illustrate this, Fig. 6 shows the distribution of route failures at different states for varying network sizes. It can be observed 


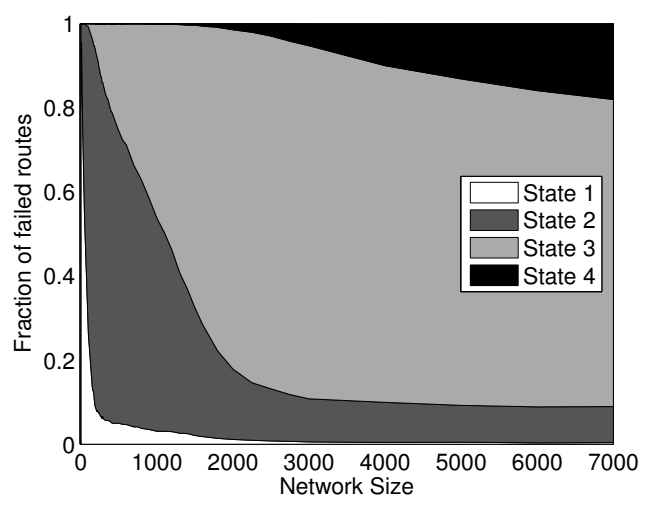

Figure 6. The Distribution of route failures as a function of states

from the figure that, for a given network size, the majority of failures occur at a single state, and that this concentration changes depending on the size of the network. For example the percentage of route failures for a network of 3,000 nodes are $<1 \%, 10 \%, 83 \%$ and $5 \%$ for states $1,2,3$ and 4 respectively. Therefore, route failure is not uniform along the states. Despite the mentioned approximations, the validation results in Fig. 5 show that the model matches well with the simulations.

\section{Stealth DHT}

\subsection{Preliminary background}

We previously proposed the Stealth DHT concept to mitigate several performance and security issues encountered in existing DHTs [1]. A Stealth DHT implementation of a given DHT algorithm creates two distinct sets of nodes with differing routing properties on the same overlay, namely Service and Stealth nodes. Service nodes provide the routing infrastructure for the overlay, whereas stealth nodes communicate with and through service nodes only.

The join process for most DHT implementations involves a node first gathering state. Usually, this is achieved by routing a join message addressed to its own ID into the DHT via a bootstrap node ${ }^{1}$. Nodes along the message's path then reply directly with relevant routing information for the joining node. Once the joining node receives notification that its message has reached its destination, it announces its presence on the network so that other nodes may route messages through it. Stealth DHTs achieve the separation of nodes by halting the join process for stealth nodes after they have gathered state, but before they announce their presence

\footnotetext{
${ }^{1}$ An already-connected node discovered through some alternate mechanism
}

on the DHT. The resultant effect is that stealth nodes do not appear in any routing tables, and thus are not used to forward any messages or store any keys.

Stealth nodes only initiate routing of messages by selecting the first hop. Therefore, they do not need to maintain a leafset which is only used to consistently determine the last hop. Stealth nodes maintain a pruned version of a routing table with only one row. This is deemed enough and has a negligible negative impact on routing performance while significantly, reducing overhead. This is because stealth nodes are only the origin of any messages they send through the DHT.

Since stealth nodes have a reduced routing table, all cells in its single row should be populated with appropriate entries. This ensures that a complete and valid routing table at a stealth node will always provide a next hop that has at least a one-digit prefix-match with the destination.

\subsection{Modelling Stealth DHT}

From the service nodes' perspective, the same model as for Pastry applies. That is, the average number of hops a lookup takes is the same as in a Pastry DHT with the network population comprised only of service nodes. In this section therefore, we need only derive the average routing performance for stealth nodes. We first derive models when only stealth nodes are considered as the origins of messages, and then present the expressions for the average number of lookup hops for the Stealth DHT with all nodes sending messages.

Observe that the first hop a stealth node makes is similar to the first hop of a service node which uses the first row of its routing table. Thus, the maximum number of lookup hops is the same as just considering a population of only service nodes. To clarify: let the fraction of service nodes be $r$ and $N$ the total number of nodes in the network, then for a Stealth DHT, $h=\log _{2^{b}} r N$.

\subsection{Perfect Routing Model}

A stealth node does not need to compare its own ID to the target key, instead, it immediately selects an appropriate node to send the message to from its routing table. Indeed, even if the stealth node does share an initial prefix match with the key, its routing table will not enable a transition to any state from state 0 other than state 1 since it has only one row in its routing table. Therefore, the transition probability from state 0 is given as:

$$
p_{i 0}= \begin{cases}0 & \text { if } i>1 \\ 1 & \text { if } i=1\end{cases}
$$

Unlike the models for a Pastry DHT, the transition from state 0 to state 1 in a Stealth DHT therefore represents the 


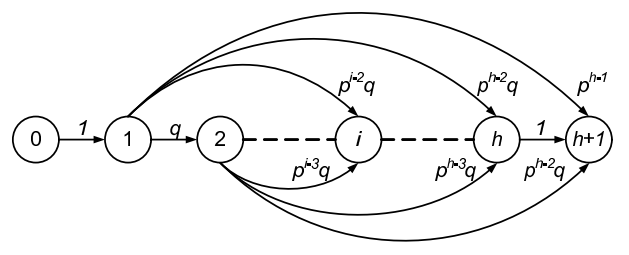

Figure 7. Markov Chain for Stealth DHT without failures

fact that a stealth node always has to use its first row of the routing table to send a message. This, together with the modified value of $h$ for Stealth DHTs, are the main distinguishing points in modelling a Stealth DHT from modelling Pastry.

The correct next hop reference in a stealth node routing table may, by chance, make as many prefix matches as possible. Thus, the Markov chain for Stealth DHT model is the same as that of Pastry model for the rest of the states. In particular, routing on a Stealth DHT is the same as routing on Pastry with a network population equal to the number of service nodes. Fig. 7 shows the Markov chain for the Stealth DHT model with perfect routing. The corresponding transition probabilities for are given as follows:

$$
p_{j i}= \begin{cases}0 & \text { if } j \leq i \\ 1 & \text { if } i=0, j=1 \\ p^{j-i-1} q & \text { if } i \geq 1, i<j \leq h \\ 1 & \text { if } i=h, j=h+1\end{cases}
$$

To obtain the expression for the average number of hops in the Stealth DHT model with perfect routing where only stealth nodes send messages $\left(H_{\text {stealth }}\right)$ and with fraction of service nodes equal to $r$ as, to modified the Markov chain the same way as we did for Pastry in Section 3. Following such Markov chain modification, the transition probability for the Stealth DHT perfect routing model is now:

$$
\mathbf{P}=\left(\begin{array}{ccccccc}
0 & 1 & 0 & 0 & \cdots 0 & 0 & 0 \\
0 & 0 & q & p q & \cdots & p^{h-2} q & p^{h-1} \\
0 & 0 & 0 & q & \cdots & p^{h-3} q & p^{h-2} \\
\cdots & \cdots & \cdots & \cdots & \cdots & \cdots & \cdots \\
0 & 0 & 0 & 0 & \cdots & q & p \\
0 & 0 & 0 & 0 & \cdots & 0 & 1 \\
1 & 0 & 0 & 0 & \cdots & 0 & 0
\end{array}\right)
$$

Note that $h=\log _{2^{b}} r N$, where $r$ is the fraction of service nodes in the network. After some arithmetic operations, we obtain the steady state solution of the corresponding linearly dependent equations as:

$$
x_{i}= \begin{cases}x_{0} & \text { if } i=1, h+1 \\ q x_{1} & \text { if } i=2 \\ x_{i-1} & \text { if } 3 \leq i \leq h\end{cases}
$$

Using this solution, we have $\pi_{h+1}=\frac{1}{(3+(h-1) q)}$, which yields the mean recurrence time for state $h+1$ of the Stealth DHT routing model $\left(E\left[T_{h+1}\right]\right)$ as :

$$
E\left[T_{h+1}\right]=3+(h-1) q
$$

Subtracting 2 from Equation (10), we obtain the average number of hops for a Stealth DHT with perfect routing and that considers only stealth nodes to send messages $\left(H_{\text {stealth }}\right)$ as:

$$
H_{\text {stealth }}=(h-1) q+1
$$

Due to the manner in which a stealth node functions, this never involves sending a message to itself (which would often be the case for small Pastry networks). The expression of the average number of hops for a Stealth DHT that considers all nodes $\left(H_{S D H T}\right)$ is:

$$
\begin{aligned}
H_{S D H T} & =r H_{\text {Pastry }}+(1-r) H_{\text {stealth }} \\
& =r h q+(1-r)[(h-1) q+1] \\
& =h q+(1-r)(1-q), r>0
\end{aligned}
$$

where $h=\log _{2^{b}} r N$ and $r$ is the fraction of service nodes.

Equation (12) shows that the average number of hops for a Stealth DHT is quite close to the average number of hops for Pastry when only service nodes are considered. This is as expected, since stealth nodes do not participate in routing and the first hop they make using their single row routing table is no different from first hop of any service node using its first row or a Pastry node in a network of the same size.

Similar to the case of Pastry, the perfect routing Stealth DHT model does not portray an entirely realistic scenario. We therefore consider a Stealth DHT model with routing imperfection in the following section.

\subsection{Model with Imperfect Routing}

The derivation of the Stealth DHT model with imperfect routing from the associated perfect routing model simply follows the same procedures as that of the equivalent Pastry models. For example, the closed form expression for transition probabilities is easily obtained as:

$$
p_{j i}= \begin{cases}0 & \text { if } j<i \\ 1 & \text { if } i=0, j=1 \\ p_{f} & \text { if } j=i, i \neq 0, h+1 \\ p^{j-i-1} q q_{f} & \text { if } i \geq 1, i<j \leq h \\ q_{f} & \text { if } i=h, j=h+1 .\end{cases}
$$

Using the same procedures as before, we get the expression for the average number lookup hops for Stealth DHT model with probability of routing failures $p_{f}$, and that considers only stealth nodes to send messages $\left(H_{\text {stealth }}^{f}\right)$ as:

$$
H_{\text {stealth }}^{f}=\frac{(h-1) q+1}{q_{f}}
$$




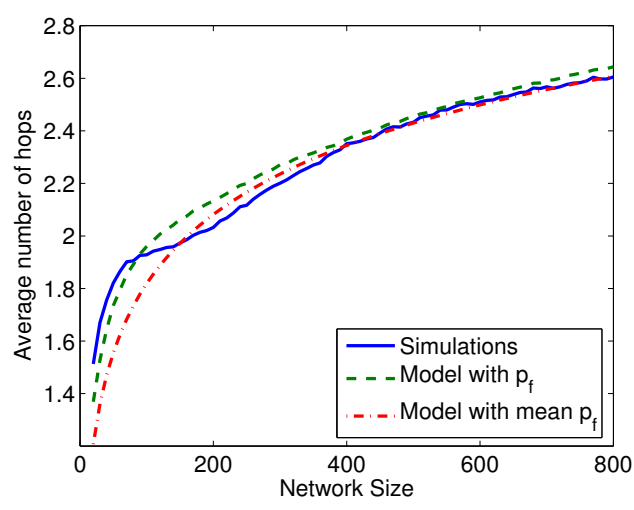

Figure 8. Validation of Stealth DHT with failures, $p_{f}=0.1093$

where $q_{f}=1-p_{f}$.

The expression of the average number of hops for a Stealth DHT with imperfect routing when considering all nodes is given as:

$$
\begin{aligned}
H_{S D H T}^{f} & =r H_{\text {Pastry }}^{f}+(1-r) H_{\text {stealth }}^{f} \\
& =\frac{h q+(1-r)(1-q)}{q_{f}}
\end{aligned}
$$

\subsection{Validation}

Simulations were carried out where 10,000 messages were sent from randomly selected stealth nodes to randomly generated IDs within the address space. A fixed network size totalling 1,000 nodes was considered, and the number of service nodes was varied from 10 to 800 , comprising $1 \%$ to $80 \%$ of the total network respectively.

Fig. 8 shows the average number of hops of a Stealth DHT obtained from models and simulations as a function of service nodes. We observe a generally excellent agreement between the model and the simulations, particularly for networks with large numbers of service nodes. However, the model underestimates the simulations for both choices of probability for route failures for small networks. This occurs in small networks because the routing imperfection in stealth nodes severely alters the routing performance by introducing randomness in choosing the next hop. Additionally since most of route failures are observed at state 1 for small networks (see Fig. 6), a route failure at a stealth node makes it very likely for the route to fail at the first service node as well. This is because the service node will also use the first row of its routing table. This, naturally makes the routing performance worse.

\section{Conclusion}

In this paper, we model the routing performance of Pastry and its corresponding Stealth DHT implementation and validate the models using simulations of the protocols. We consider a perfect routing case in which nodes' routing tables are assumed to be always full, as well a realistic case where some cells in routing tables are often empty. Routing table imperfection causes a lookup to follow a non-optimal routing path, which, through the derived models in this paper, is shown to have an exponentially negative effect on the routing performance of the protocols. We use simulations to demonstrate that the models offer average routing performance that agree with the simulation results very well. They can therefore be used instead of simulations to quickly evaluate the protocols in a wide variety of experimental setups.

\section{References}

[1] A. Brampton, A. MacQuire, I. A. Rai, N. J. P. Race, and L. Mathy. Stealth Distributed Hash Table: A robust and flexible super-peered DHT. In Proc. of the 2nd Conference on Future Networking Technologies (CoNEXT), Lisbon, Portugal, December 2006.

[2] K. L. Calvert, M. B. Doar, and E. W. Zegura. Modeling Internet topology. IEEE Communications Magazine, 35(6):160163, June 1997.

[3] M. F. Kaashoek and D. R. Karger. Koorde: A simple degreeoptimal distributed hash table. In Proc. of the 2nd International Workshop on Peer-to-Peer Systems (IPTPS'03), 2003.

[4] A. Leon-Garcia. Probability and Random Processes for Electrical Engineering. Addison-Wesley, second edition, 1994.

[5] S. Ratnasamy, P. Francis, M. Handley, R. Karp, and S. Shenker. A scalable content-addressable network. In Proc. of ACM SIGCOMM, August 2001.

[6] A. Rowstron and P. Druschel. Pastry: Scalable, distributed object location and routing for large-scale peer-to-peer systems. In Proc. of the 18th IFIP/ACM International Conference on Distributed Systems Platforms (Middleware), Heidelberg, Germany, November 2001.

[7] A. Spognardi and R. D. Pietro. A formal framework for the performance analysis of P2P networks protocols. In Proc. of the 3rd International Workshop on Hot Topics in Peer-toPeer Systems (Hot-P2P), Rhodes Island, Greece, April 2006.

[8] A. Spognardi, R. D. Pietro, and L. V. Mancini. BaRT, balanced randomized tree: A scalable and distributed protocol for lookup in peer-to-peer networks. In Proc. of the 1st International Workshop on Hot Topics in Peer-to-Peer Systems (Hot-P2P'04), 2004.

[9] I. Stoica, R. Morris, D. Karger, F. Kaashoek, and H. Balakrishnan. Chord: A scalable peer-to-peer lookup service for Internet applications. In Proc. of ACM SIGCOMM, pages 149-160, August 2001

[10] B. Y. Zhao, L. Huang, J. Stribling, S. C. Rhea, A. D. Joseph, and J. Kubiatowicz. Tapestry: A resilient global-scale overlay for service deployment. IEEE Journal on Selected Areas in Communications, 22(1):41-53, 2004. 